\title{
Use of Diatomaceous Earth as a Silica Supplement on Potted Ornamentals
}

\author{
Taylor Mills-Ibibofori ${ }^{1}$, , Bruce Dunn ${ }^{1, *}$, Niels Maness ${ }^{1}$ and Mark Payton ${ }^{2}$ \\ 1 Department of Horticulture \& L.A., Oklahoma State University, Stillwater, OK 74078, USA; \\ tsmills@okstate.edu (T.M.-I.); niels.maness@okstate.edu (N.M.) \\ 2 Department of Statistics, Oklahoma State University, Stillwater, OK 74078, USA; mark.payton@okstate.edu \\ * Correspondence: bruce.dunn@okstate.edu
}

Received: 28 January 2019; Accepted: 22 February 2019; Published: 1 March 2019

check for updates

\begin{abstract}
The role of silica as a needed supplement in soilless media is gaining interest. This research studied the effects of diatomaceous earth as a supplement on growth and flower characteristics, physiology, and nutrient uptake in dahlia (Dahlia Cav. $\times$ hybrida 'Dahlinova Montana'), black-eyed Susan (Rudbeckia hirta L. 'Denver Daisy'), and daisy (Gerbera jamesonii L. 'Festival Light Eye White Shades'). Plants were either well-watered at 10 centibars or water-stressed at 20 centibars. Silicon treatments included top-dressed at 20,40,60, and $80 \mathrm{~g}$, or incorporated at 50, 100, 150, and $200 \mathrm{~g}$, in Metro-Mix 360 media without silica plus a control and one treatment of new Metro-Mix 360 with silica already incorporated. Significant effects were seen from diatomaceous earth supplementation, irrigation, and interaction in all plants; growth and flower characteristics, leaf nutrient content, and tolerance to stress were improved by application of diatomaceous earth. An increase in leaf N, P, $\mathrm{K}, \mathrm{Mg}$, and Ca was observed for dahlia 'Dahlinova Montana' and black-eyed Susan 'Denver Daisy'. Transpiration was maintained in all three species due to silica supplementation under water-stress. Metro-Mix with silica was similar to the Metro-mix without silica and equivalent to most treatments with supplemental silica for all three species.
\end{abstract}

Keywords: greenhouse; metro-mix; Dahlia; Rudbeckia; Gerbera

\section{Introduction}

Silicon is the second most abundant element on earth and is present in various forms, including silicon dioxide, also known as silica ( $\mathrm{Si}$ ). In plants, except for members of the family Equisetaceae, $\mathrm{Si}$ is a nonessential and beneficial element, meaning that plants can complete their life cycles without the mineral nutrient [1]. However, plants deficient in Si are often weaker structurally and more prone to abnormalities of growth, development, and reproduction. The benefits of $\mathrm{Si}$ are mostly evident when plants are under stress conditions [2]. Several studies have shown that plants benefit in many ways from supplemental soluble $\mathrm{Si}$, including greater tolerance of environmental stresses, drought, salinity, mineral toxicity or deficiency, improved growth rates, and resistance to insects and fungi [3-5].

Common use of soilless substrates in greenhouse and nursery production limits the availability of Si to plants [6]. Plants grown with soilless media often appear weaker structurally compared to crops grown in the field [7]. Therefore, adding Si-related compounds as an amendment has been highly recommended. Miyake and Takahashi [8] brought interest to Si nutrition of horticultural crops when they observed Si deficient tomatoes (Lycopersicon esculentum Mill.). In the Netherlands, the use of Si supplementation in a hydroponic system was recommended for crops such as cucumber (Cucumus sativus L.) and roses (Rosa hybrida L.) $[9,10]$. Roses with Si added to the nutrient formula also showed a decrease in leaf and flower senescence [11]. The shelf life of Chrysanthemum L. cut flowers was also 
extended [12]. Hydroponically-produced gerbera plants supplemented with Si had improved overall crop and flower quality [13].

Other considerations such as solubility, availability, physical properties, and contaminants must be considered before choosing a Si source. Silica is available from natural resources, fertilizers (organic and inorganic), and industrial by-products. Most horticultural studies use Si from by-products such as liquid silicates, slag, and basalt dust [14-16]. Diatomaceous earth (DE) is a sedimentary rock formed from the deposition of silica-rich diatoms. The cell walls of diatoms contain amorphous silica $\left(\mathrm{SiO}_{2} \cdot \mathrm{H}_{2} \mathrm{O}\right)$. There has been limited research focused on the effects of $\mathrm{DE}$ regarding growth and flower characteristics, as well as water-stress related issues in horticultural crops. Most studies utilizing DE focused on retention of water or circulation of oxygen in plant media [17]. However, supplementation of DE has been proven to improve plant growth, quality, and nutrient uptake in agronomic crops, such as rice (Oryza sativa L.) [18]. Use of DE to improve plant growth of ornamentals is limited, thus the objectives of this study were to determine the effects of DE as a Si supplement on three potted ornamentals under well-watered and water-stressed conditions.

\section{Materials and Methods}

\subsection{Plant Material and Culture}

On 8 May 2015, two 128 plug cell trays of black-eyed Susan (Rudbeckia hirta L. 'Denver Daisy'), five 51 plug cell trays of dahlia (Dahlia Cav. × hybrida 'Dahlinova Montana'), and two 128 plug cell trays of daisy (Gerbera jamesonii L. 'Festival Light Eye White Shades') were obtained from Park Seed (Greenwood, SC, USA). Before transplanting, all species were placed on a mist bench. Cuttings and plugs were transplanted into standard $15 \mathrm{~cm}$ pots, filled with media (Metro-Mix (MM) 360; Sun Gro Horticulture, Bellevue, WA, USA) that did not contain Si on 28 May 2015, and a single treatment of media (MM + Si; Sun Gro Horticulture, Bellevue, WA, USA) that contained 20 to $50 \mathrm{ppm}$ soluble Si (RESiLIENCE ${ }^{\mathrm{TM}}$ ) derived from wollastonite [19]. A single plant was placed in each pot and plants were grown at the Department of Horticulture and L.A. research greenhouses in Stillwater, OK under natural photoperiods. Temperatures were set at $37^{\circ} \mathrm{C}$ during the day and $26{ }^{\circ} \mathrm{C}$ during the night.

\subsection{Experimental Arrangement}

Ten Si treatments were established by adding diatomaceous earth (Perma-Guard, Inc., Kamas, UT, USA). Application of DE included top-dressed (TD) rates at 20, 40,60, and $80 \mathrm{~g}$, and incorporated (INC) rates at 50, 100, 150, and $200 \mathrm{~g}$. An MM control (using media without DE) and the MM + Si treatments were also included. For each species, there were six pots per Si treatment per irrigation treatment, which served as single pot replicates. Plants were well-watered at 10 centibars or water-stressed at 20 centibars using one drip emitter per pot. Tensiometers (IRROMETER, Riverside, CA, USA) were used to control irrigation by placing a single tensiometer in the middle of a bench in a pot at a depth of $10 \mathrm{~cm}$, which resulted in no leachate. Plant species and Si treatments were randomized within irrigation, which served as blocks.

\subsection{Harvesting and Measurements}

Data collected on plants included height from the media surface to the tallest opened flower, width (average of two perpendicular measurements), shoot dry weight, number of flowers, flower diameter, and transpiration. Shoot dry weight was determined by cutting the stems at media level and drying for $2 \mathrm{~d}$ at $52.2^{\circ} \mathrm{C}$. For foliar nutrient analyses, mature leaves from the middle to upper level of the plant were collected from five plants per Si and irrigation treatment of each species. Soil and leaf nutrient analysis was performed by the Soil, Water, and Forage Analytical Laboratory (SWFAL) at Oklahoma State University, using a LECO TruSpec Carbon and Nitrogen Analyzer (LECO Corporation, St. Joseph, MI, USA). Soil and leaf Si analysis was performed, using the $0.5 \mathrm{M}$ ammonium acetate 
method [20]. Transpiration was recorded weekly using a LI-1600 Steady State Porometer (LI-COR Inc., Lincoln, NE, USA).

\subsection{Statistical Analysis}

Pots were arranged in a randomized block design with irrigation serving as the block. Analysis of variance methods (PROC MIXED) were used with a two-factor factorial arrangement, with irrigation and silicon treatment as the factors of interest. Separate analyses were conducted for each of the plant species. When interactions of irrigation and Si treatment were significant, simple effects were reported. Mean separations were determined using a DIFF option in an LSMEANS statement and a SLICE option (when appropriate) and with a 0.05 level of significance.

\section{Results}

\subsection{Dahlia xhybrida 'Dahlinova Montana'}

A significant interaction of $\mathrm{Si}$ treatment with irrigation was seen for transpiration and soil $\mathrm{Si}$ (Table 1). Under the well-watered condition, soil Si was greatest when supplemented with 60 and $80 \mathrm{~g}$ TD, as well as 100, 150, and $200 \mathrm{~g}$ INC (Table 2). Transpiration was greatest in plants under the control as well as 40 and $60 \mathrm{~g}$ TD. Under the water-stressed condition, soil Si was greatest for INC plants compared to TD plants and MM + Si plants. Plants treated with $100 \mathrm{~g}$ or less DE within the INC treatment had the greatest transpiration.

Table 1. Analysis of variance for growth, flowering, leaf nutrient content, soil silica, and physiology of Dahlia $\times$ hybrida 'Dahlinova Montana' after application of diatomaceous earth (DE) and irrigation controlled with a tensiometer.

\begin{tabular}{|c|c|c|c|c|c|c|c|c|c|c|c|c|c|}
\hline Source & \multicolumn{3}{|c|}{$\begin{array}{l}\text { Height } \\
\text { (cm) }\end{array}$} & \multicolumn{2}{|c|}{$\begin{array}{l}\text { Width } \\
\text { (cm) }\end{array}$} & \multicolumn{2}{|c|}{$\begin{array}{l}\text { Shoot Dry } \\
\text { Weight } \\
\text { (g) }\end{array}$} & \multicolumn{2}{|c|}{$\begin{array}{c}\text { Stem } \\
\text { Diameter } \\
(\mathrm{cm})\end{array}$} & \multicolumn{2}{|c|}{$\begin{array}{l}\text { Mean Flower } \\
\text { Number }\end{array}$} & \multicolumn{2}{|c|}{$\begin{array}{c}\text { Flower } \\
\text { Diameter } \\
(\mathrm{cm})\end{array}$} \\
\hline Irrigation & \multicolumn{3}{|c|}{$* * * *$} & \multicolumn{2}{|c|}{$* * * * *$} & \multicolumn{2}{|c|}{$* * * * *$} & \multicolumn{2}{|c|}{$* * *$} & \multicolumn{2}{|c|}{$* * * *$} & \multicolumn{2}{|c|}{$* * * *$} \\
\hline $\begin{array}{l}\text { DE Treatment } \\
\times \text { Irrigation }\end{array}$ & \multicolumn{3}{|c|}{ ns } & \multicolumn{2}{|c|}{ ns } & \multicolumn{2}{|c|}{ ns } & \multicolumn{2}{|c|}{ ns } & \multicolumn{2}{|c|}{ ns } & \multicolumn{2}{|c|}{ ns } \\
\hline DE Treatment & $* *$ & * & * & $\mathrm{ns}$ & $* * *$ & $* * * *$ & ns & $* * * *$ & $\mathrm{~ns}$ & $* * *$ & $* *$ & $* * * *$ & ns \\
\hline Irrigation & ns & ns & ns & ns & ns & ns & ns & ns & ns & ns & $* * *$ & ns & ns \\
\hline $\begin{array}{l}\text { DE Treatment } \\
\times \text { Irrigation }\end{array}$ & ns & ns & ns & ns & ns & ns & ns & ns & ns & ns & ns & ns & ns \\
\hline \multicolumn{4}{|c|}{ DE Treatment $\times$ Irrigation } & \multicolumn{4}{|c|}{$* * * *$} & & & \multicolumn{2}{|r|}{$* *$} & & \\
\hline
\end{tabular}

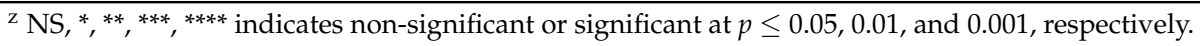

A significant effect of irrigation was seen for all growth and flowering characteristics (Table 1). Well-watered plants had greater height, width, shoot dry weight, mean flower number, stem diameter, and flower diameter, compared to water-stressed plants (Table 3). A significant effect of DE treatment was seen for height, shoot dry weight, and stem diameter (Table 1). Height was greatest for control plants, all TD plants, INC plants at 100 and $200 \mathrm{~g}$, as well as MM + Si plants (Table 4). Shoot dry weight was greatest for control plants, all TD plants, and INC plants at $100 \mathrm{~g}$. Stem diameter was greatest for all TD plants, as well as INC plants at 50 and $100 \mathrm{~g}$. 
Table 2. Soil silica $(\mathrm{Si})$, transpiration, and leaf $\mathrm{Si}$ affected by interaction of diatomaceous earth treatment with irrigation in Dahlia $\times$ hybrida 'Dahlinova Montana', Gerbera jamesonii 'Festival Light Eye White Shades', and Rudbeckia hirta 'Denver Daisy'.

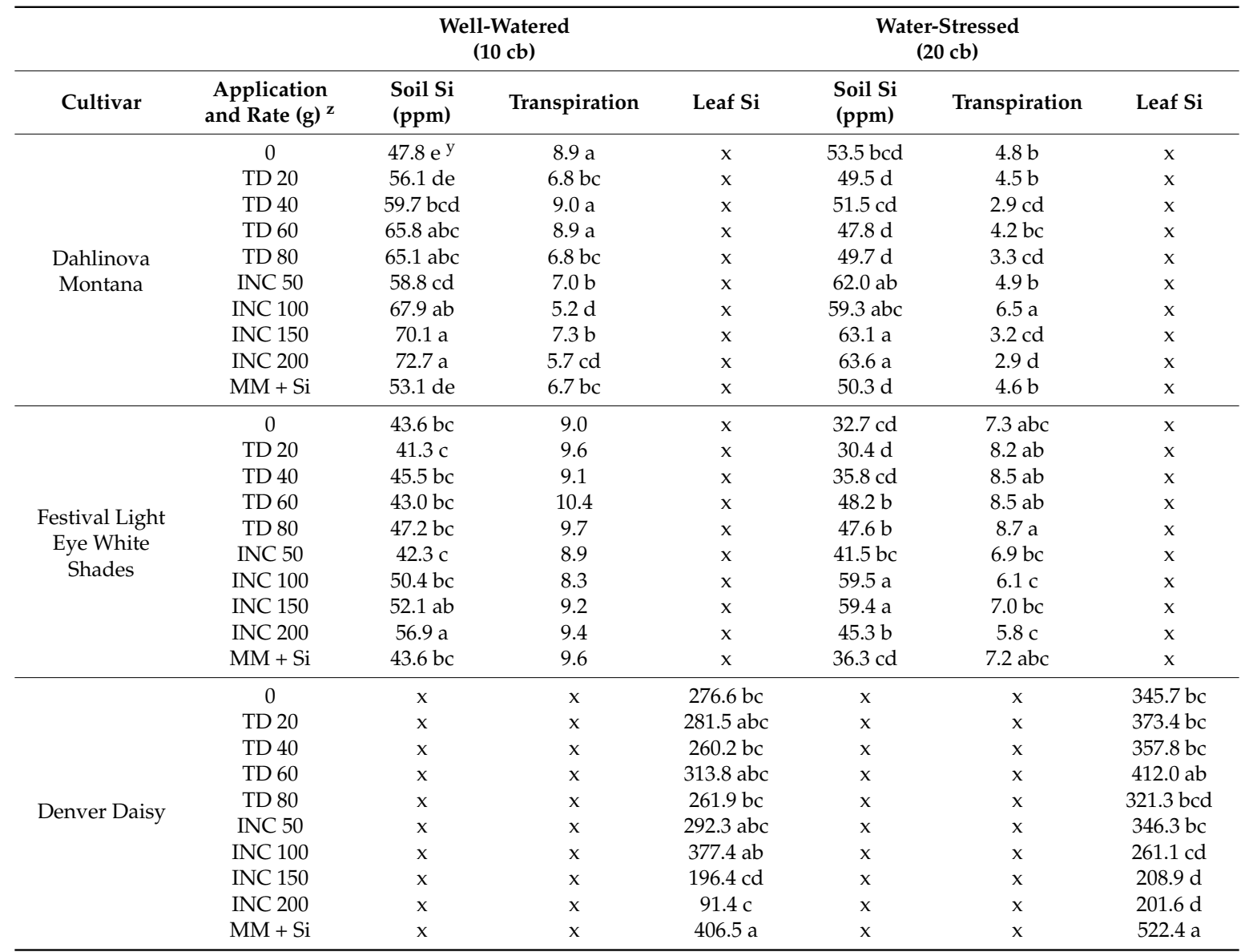

${ }^{\mathrm{z}}$ Top-dressed (TD), Incorporated (INC), and Metro-Mix media (MM). ${ }^{y}$ Means $(n=6)$ with the same letter within the same column and within cultivar are not statistically different at $p \leq 0.05 .{ }^{\times}$Main effects were significant for factors.

Table 3. Growth and flowering characteristics affected by irrigation, controlled by a tensiometer, averaged across diatomaceous earth treatments in Dahlia $\times$ hybrida 'Dahlinova Montana', Gerbera jamesonii 'Festival Light Eye White Shades', and Rudbeckia hirta 'Denver Daisy'.

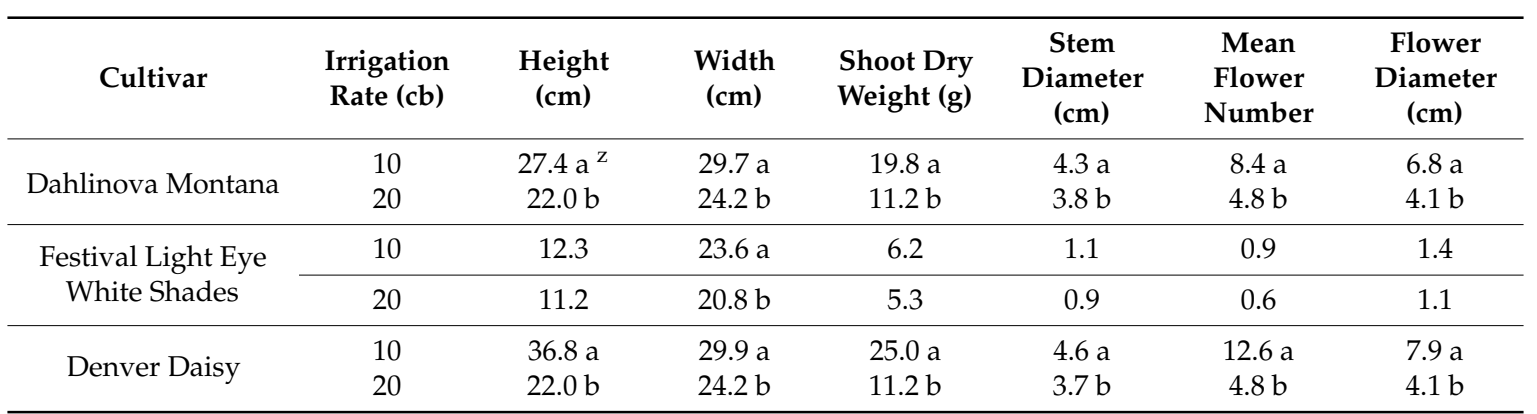

${ }^{\mathrm{z}}$ Means $(\mathrm{n}=6)$ with the same letter within the same column and within cultivar are not statistically significant at $p \leq 0.05$. 
Table 4. Growth and flowering characteristics affected by diatomaceous earth treatment averaged across irrigation, controlled by a tensiometer, in Dahlia $\times$ hybrida 'Dahlinova Montana', Gerbera jamesonii 'Festival Light Eye White Shades', and Rudbeckia hirta 'Denver Daisy'.

\begin{tabular}{|c|c|c|c|c|c|c|c|}
\hline Cultivar & $\begin{array}{l}\text { Application } \\
\text { and Rate } \\
\text { (g) } \mathrm{z}\end{array}$ & $\begin{array}{l}\text { Height } \\
\text { (cm) }\end{array}$ & $\begin{array}{l}\text { Width } \\
\text { (cm) }\end{array}$ & $\begin{array}{l}\text { Shoot Dry } \\
\text { Weight } \\
\text { (g) }\end{array}$ & $\begin{array}{c}\text { Stem } \\
\text { Diameter } \\
\text { (cm) }\end{array}$ & $\begin{array}{c}\text { Mean } \\
\text { Flower } \\
\text { Number }\end{array}$ & $\begin{array}{c}\text { Flower } \\
\text { Diameter } \\
\quad(\mathrm{cm})\end{array}$ \\
\hline \multirow{10}{*}{$\begin{array}{c}\text { Dahlinova } \\
\text { Montana }\end{array}$} & 0 & $24.9 \mathrm{ab}^{\mathrm{y}}$ & 26.9 & $15.8 \mathrm{a}-\mathrm{d}$ & $3.8 \mathrm{bc}$ & 7.1 & 5.7 \\
\hline & TD 20 & $24.1 \mathrm{ab}$ & 29.1 & 18.9 a & $4.5 \mathrm{a}$ & 8.0 & 5.8 \\
\hline & TD 40 & $24.8 \mathrm{ab}$ & 27.9 & $19.0 \mathrm{a}$ & $4.5 \mathrm{a}$ & 8.5 & 5.4 \\
\hline & TD 60 & $26.9 \mathrm{a}$ & 29.3 & $17.9 \mathrm{ab}$ & $4.3 \mathrm{ab}$ & 5.7 & 4.9 \\
\hline & TD 80 & $26.8 \mathrm{a}$ & 26.9 & $16.2 \mathrm{abc}$ & $4.5 \mathrm{a}$ & 7.0 & 5.3 \\
\hline & INC 50 & $22.2 \mathrm{~b}$ & 24.3 & $14.5 \mathrm{bcd}$ & $3.9 \mathrm{ac}$ & 5.9 & 4.9 \\
\hline & INC 100 & $26.6 \mathrm{a}$ & 27.5 & $15.2 \mathrm{a}-\mathrm{d}$ & $4.3 \mathrm{ab}$ & 6.3 & 6.3 \\
\hline & INC 150 & $22.1 \mathrm{~b}$ & 25.9 & $11.8 \mathrm{~d}$ & $3.4 \mathrm{c}$ & 5.4 & 5.3 \\
\hline & INC 200 & $23.9 \mathrm{ab}$ & 25.1 & $12.6 \mathrm{~cd}$ & $3.4 \mathrm{c}$ & 5.5 & 5.4 \\
\hline & $\mathrm{MM}+\mathrm{Si}$ & $24.9 \mathrm{ab}$ & 27.4 & $13.1 \mathrm{~cd}$ & $3.8 \mathrm{bc}$ & 6.4 & 5.4 \\
\hline \multirow{10}{*}{$\begin{array}{l}\text { Festival } \\
\text { Light Eye } \\
\text { White } \\
\text { Shades }\end{array}$} & 0 & $8.9 \mathrm{~b}^{\mathrm{y}}$ & $20.8 \mathrm{~b}$ & $3.9 c$ & 0.9 & 0.4 & 0.6 \\
\hline & TD 20 & $11.1 \mathrm{~b}$ & $21.7 \mathrm{ab}$ & $5.9 \mathrm{bc}$ & 1.2 & 1.1 & 2.1 \\
\hline & TD 40 & $11.2 \mathrm{~b}$ & $22.5 \mathrm{ab}$ & $4.9 \mathrm{bc}$ & 0.7 & 0.5 & 1.1 \\
\hline & TD 60 & $10.4 \mathrm{~b}$ & $22.4 \mathrm{ab}$ & $5.4 \mathrm{bc}$ & 0.6 & 0.3 & 0.2 \\
\hline & TD 80 & $11.8 \mathrm{ab}$ & $20.0 \mathrm{~b}$ & $5.9 \mathrm{bc}$ & 0.3 & 0.8 & 1.2 \\
\hline & INC 50 & $12.1 \mathrm{ab}$ & $21.6 \mathrm{ab}$ & $5.7 \mathrm{bc}$ & 1.3 & 0.6 & 1.0 \\
\hline & INC 100 & $15.1 \mathrm{a}$ & $25.2 \mathrm{a}$ & $8.6 \mathrm{a}$ & 1.5 & 1.2 & 2.8 \\
\hline & INC 150 & $15.3 \mathrm{a}$ & $24.9 \mathrm{a}$ & $7.2 \mathrm{ab}$ & 1.5 & 1.0 & 2.1 \\
\hline & INC 200 & $10.8 \mathrm{~b}$ & $23.1 \mathrm{ab}$ & $5.0 \mathrm{bc}$ & 1.5 & 1.0 & 1.6 \\
\hline & $\mathrm{MM}+\mathrm{Si}$ & $10.8 \mathrm{~b}$ & $19.5 \mathrm{~b}$ & $4.9 \mathrm{bc}$ & 0.8 & 0.8 & 0.6 \\
\hline \multirow{10}{*}{$\begin{array}{c}\text { Denver } \\
\text { Daisy }\end{array}$} & 0 & $30.8 \mathrm{bc}{ }^{\mathrm{y}}$ & $23.0 \mathrm{bc}$ & 12.8 & 4.3 & 6.4 & $6.2 \mathrm{~b}$ \\
\hline & TD 20 & $31.1 \mathrm{bc}$ & $26.9 \mathrm{ab}$ & 17.6 & 4.4 & 10.5 & $5.4 \mathrm{bc}$ \\
\hline & TD 40 & 30.9 bc & $26.9 \mathrm{ab}$ & 18.1 & 3.9 & 9.8 & $5.5 \mathrm{bc}$ \\
\hline & TD 60 & $34.8 \mathrm{abc}$ & $27.2 \mathrm{ab}$ & 23.4 & 4.7 & 11.1 & $5.9 \mathrm{bc}$ \\
\hline & TD 80 & $30.9 \mathrm{bc}$ & $28.2 \mathrm{a}$ & 24.2 & 4.5 & 9.7 & $6.4 \mathrm{~b}$ \\
\hline & INC 50 & $32.2 \mathrm{bc}$ & $26.1 \mathrm{abc}$ & 18.2 & 4.2 & 9.6 & $6.5 \mathrm{ab}$ \\
\hline & INC 100 & $46.4 \mathrm{a}$ & $27.8 \mathrm{a}$ & 19.9 & 3.8 & 8.9 & $5.9 \mathrm{bc}$ \\
\hline & INC 150 & $25.3 c$ & $21.9 c$ & 15.5 & 4.0 & 7.0 & $4.9 \mathrm{bc}$ \\
\hline & INC 200 & $27.4 \mathrm{bc}$ & $23.1 \mathrm{bc}$ & 16.1 & 3.7 & 8.5 & $4.5 \mathrm{a}$ \\
\hline & $\mathrm{MM}+\mathrm{Si}$ & $39.2 \mathrm{ab}$ & $29.2 \mathrm{a}$ & 20.2 & 4.3 & 11.1 & $8.1 \mathrm{a}$ \\
\hline
\end{tabular}

${ }^{\mathrm{z}}$ Top-dressed (TD), Incorporated (INC), and Metro-Mix media (MM). ${ }^{y}$ Means $(n=6)$ with the same letter within the same column and within cultivar are not statistically different at $p \leq 0.05$.

A significant effect of DE treatment was seen for leaf nutrient content (Table 1). Total nitrogen $(\mathrm{N})$ was greatest for all TD plants and INC plants with rates of 100, 150, and $200 \mathrm{~g}$ (Table 5). TD plants at $80 \mathrm{~g}$ and INC plants at 100, 150, and $200 \mathrm{~g}$ had the greatest values of phosphorus (P). Magnesium $(\mathrm{Mg})$ was greatest for TD plants at 40 and $80 \mathrm{~g}$, as well as INC plants at 50, 100, and $150 \mathrm{~g}$. Calcium (Ca) was greatest for INC plants at 50, 100, and $150 \mathrm{~g}$, as well as MM + Si plants. Sulfur (S) was greatest for all TD plants and INC plants at 100, 150, and $200 \mathrm{~g}$ (Table 6). Silica was greatest for control plants, TD plants at 20, 40, and $60 \mathrm{~g}$, INC plants at 100 and $150 \mathrm{~g}$, as well as MM + Si plants. For copper $(\mathrm{Cu})$, the greatest values were seen for TD plants at 40 and $80 \mathrm{~g}$. Iron (Fe) was greatest for TD plants at 40, 60, and $80 \mathrm{~g}$, as well as INC plants at 100 and $150 \mathrm{~g}$. Manganese (Mn) was greatest for INC plants at 150 and $200 \mathrm{~g}$. 
Table 5. Leaf macronutrient content affected by diatomaceous earth treatment across irrigation, controlled by a tensiometer, in Dahlia $\times$ hybrida 'Dahlinova Montana', Gerbera jamesonii 'Festival Light Eye White Shades', and Rudbeckia hirta 'Denver Daisy'.

\begin{tabular}{|c|c|c|c|c|c|c|}
\hline Cultivar & $\begin{array}{l}\text { Application } \\
\text { and Rate } \\
\text { (g) } z\end{array}$ & N (\%) & $P(\%)$ & K (\%) & $\operatorname{Mg}(\%)$ & $\mathrm{Ca}(\%)$ \\
\hline \multirow{10}{*}{$\begin{array}{l}\text { Dahlinova } \\
\text { Montana }\end{array}$} & 0 & $3.55 \mathrm{bcd}^{\mathrm{y}}$ & $0.29 \mathrm{c}$ & 3.51 & $0.92 \mathrm{bc}$ & $1.74 \mathrm{de}$ \\
\hline & TD 20 & $3.88 \mathrm{ab}$ & $0.33 \mathrm{bc}$ & 3.33 & $0.91 \mathrm{bc}$ & $1.81 \mathrm{~cd}$ \\
\hline & TD 40 & $4.11 \mathrm{ab}$ & $0.34 \mathrm{bc}$ & 3.19 & $0.97 \mathrm{ab}$ & $1.75 \mathrm{de}$ \\
\hline & TD 60 & $4.09 \mathrm{ab}$ & $0.33 \mathrm{bc}$ & 3.23 & $0.84 \mathrm{c}$ & $1.61 \mathrm{e}$ \\
\hline & TD 80 & $3.83 \mathrm{ab}$ & $0.34 \mathrm{abc}$ & 3.47 & $0.96 \mathrm{ab}$ & $1.85 \mathrm{bcd}$ \\
\hline & INC 50 & $3.21 \mathrm{~cd}$ & $0.29 c$ & 3.48 & $0.98 \mathrm{ab}$ & $1.93 \mathrm{abc}$ \\
\hline & INC 100 & $3.75 \mathrm{abcd}$ & $0.35 a b c$ & 3.54 & $1.03 \mathrm{a}$ & $1.99 \mathrm{ab}$ \\
\hline & INC 150 & $3.79 \mathrm{abc}$ & $0.38 \mathrm{ab}$ & 3.81 & $0.98 \mathrm{ab}$ & $2.00 \mathrm{ab}$ \\
\hline & INC 200 & $4.27 \mathrm{a}$ & $0.39 a$ & 3.54 & $0.92 \mathrm{bc}$ & $1.88 \mathrm{bcd}$ \\
\hline & $\mathrm{MM}+\mathrm{Si}$ & $3.19 \mathrm{~d}$ & $0.33 \mathrm{bc}$ & 3.44 & $0.82 \mathrm{c}$ & $2.07 \mathrm{a}$ \\
\hline \multirow{10}{*}{$\begin{array}{c}\text { Festival Light } \\
\text { Eye White } \\
\text { Shades }\end{array}$} & 0 & $2.86^{\mathrm{y}}$ & 0.26 & 2.63 & 0.62 & 1.45 \\
\hline & TD 20 & 2.92 & 0.31 & 3.18 & 0.66 & 1.55 \\
\hline & TD 40 & 3.05 & 0.28 & 3.30 & 0.62 & 1.48 \\
\hline & TD 60 & 2.93 & 0.46 & 3.06 & 0.77 & 2.12 \\
\hline & TD 80 & 3.14 & 0.54 & 3.31 & 0.79 & 2.02 \\
\hline & INC 50 & 2.74 & 0.29 & 3.10 & 0.73 & 1.71 \\
\hline & INC 100 & 2.82 & 0.27 & 3.06 & 0.62 & 1.42 \\
\hline & INC 150 & 2.95 & 0.26 & 3.03 & 0.55 & 1.26 \\
\hline & INC 200 & 2.98 & 0.26 & 2.97 & 0.56 & 1.33 \\
\hline & $\mathrm{MM}+\mathrm{Si}$ & 2.99 & 0.37 & 3.10 & 0.70 & 1.88 \\
\hline \multirow{10}{*}{ Denver Daisy } & 0 & $2.40 \mathrm{de}^{\mathrm{y}}$ & $0.20 \mathrm{e}$ & $3.09 \mathrm{~d}$ & $1.21 \mathrm{a}$ & $3.35 \mathrm{~b}$ \\
\hline & TD 20 & 2.79 cde & 0.22 cde & $3.31 \mathrm{bcd}$ & $1.20 \mathrm{ab}$ & $3.33 \mathrm{~b}$ \\
\hline & TD 40 & $2.98 \mathrm{bc}$ & $0.26 \mathrm{bcd}$ & $3.53 \mathrm{bcd}$ & $1.10 \mathrm{ab}$ & $2.89 \mathrm{bc}$ \\
\hline & TD 60 & $2.94 \mathrm{bc}$ & 0.23 cde & $3.25 \mathrm{~cd}$ & $1.13 \mathrm{ab}$ & $3.28 \mathrm{bc}$ \\
\hline & TD 80 & $2.87 \mathrm{~cd}$ & $0.21 \mathrm{de}$ & $3.29 \mathrm{bcd}$ & $1.12 \mathrm{ab}$ & $3.17 \mathrm{bc}$ \\
\hline & INC 50 & 2.82 cde & $0.24 \mathrm{~b}-\mathrm{e}$ & $3.27 \mathrm{bcd}$ & $1.15 \mathrm{ab}$ & $3.27 \mathrm{bc}$ \\
\hline & INC 100 & $2.96 \mathrm{bc}$ & $0.27 \mathrm{abc}$ & $3.74 \mathrm{ab}$ & $1.12 \mathrm{ab}$ & $3.26 \mathrm{bc}$ \\
\hline & INC 150 & $3.38 \mathrm{ab}$ & $0.28 \mathrm{ab}$ & $3.72 \mathrm{abc}$ & $1.09 \mathrm{bc}$ & $3.26 \mathrm{bc}$ \\
\hline & INC 200 & $3.51 \mathrm{a}$ & $0.31 \mathrm{a}$ & $4.13 \mathrm{a}$ & $0.97 \mathrm{~cd}$ & $2.79 \mathrm{c}$ \\
\hline & $\mathrm{MM}+\mathrm{Si}$ & $2.37 \mathrm{e}$ & $0.21 \mathrm{de}$ & $3.14 \mathrm{~d}$ & $0.96 \mathrm{~d}$ & $4.01 \mathrm{a}$ \\
\hline \multicolumn{2}{|c|}{ Optimum levels $\mathrm{x}$} & $2.50-4.50$ & $0.20-0.75$ & $1.50-5.50$ & $0.25-1.00$ & $1.00-4.00$ \\
\hline
\end{tabular}

${ }^{\mathrm{z}}$ Top-dressed (TD), incorporated (INC), and Metro-Mix media (MM). ${ }^{\mathrm{y}}$ Means ( $\mathrm{n}=6$ ) with the same letter within the same column and within cultivars are not statistically significant at $p \leq 0.05 .{ }^{\times}$According to Kalra (26).

\subsection{Gerbera jamesonii 'Festival Light Eye White Shades'}

A significant interaction of DE with irrigation was seen for soil Si and transpiration (Table 7). Under the well-watered condition, soil Si was greatest when DE was supplemented at 150 and $200 \mathrm{~g}$ INC (Table 2). Under the water-stressed condition, soil Si was greatest for 100 and $150 \mathrm{~g}$ INC plants. Transpiration was greatest for control plants, TD plants, and MM + Si plants. A significant effect of irrigation was seen for width and leaf nutrient content (Table 7). Well-watered plants had greater widths compared to water-stressed plants (Table 3). Potassium, $\mathrm{Ca}$, sodium ( $\mathrm{Na}$ ), and $\mathrm{Mn}$ levels were greater in water-stressed plants compared to well-watered plants (Table 8). 
Table 6. Leaf micronutrient and trace element content affected by diatomaceous earth treatment across irrigation, controlled by a tensiometer, in Dahlia $\times$ hybrida 'Dahlinova Montana', Gerbera jamesonii 'Festival Light Eye White Shades', and Rudbeckia hirta 'Denver Daisy'.

\begin{tabular}{|c|c|c|c|c|c|c|c|c|c|}
\hline Cultivar & $\begin{array}{c}\text { Application } \\
\text { and Rate }(g)^{z}\end{array}$ & $\begin{array}{c}\mathrm{S} \\
(\%)\end{array}$ & $\begin{array}{l}\mathrm{Na} \\
(\%)\end{array}$ & $\begin{array}{c}\mathrm{Si} \\
(\mathrm{ppm})\end{array}$ & $\begin{array}{c}\mathrm{Zn} \\
(\mathrm{ppm})\end{array}$ & $\begin{array}{c}\mathrm{Cu} \\
(\mathrm{ppm})\end{array}$ & $\begin{array}{c}\text { Mn } \\
(\mathrm{ppm})\end{array}$ & $\begin{array}{c}\mathrm{Ni} \\
(\mathrm{ppm})\end{array}$ & $\begin{array}{c}\text { Fe } \\
\text { (ppm) }\end{array}$ \\
\hline \multirow{10}{*}{$\begin{array}{l}\text { Dahlinova } \\
\text { Montana }\end{array}$} & 0 & $0.33 b c^{y}$ & 0.02 & $84.1 \mathrm{a}$ & $38.5 \mathrm{a}$ & $12.0 \mathrm{~cd}$ & $151.6 \mathrm{~d}$ & 0.0 & $92.2 \mathrm{~d}$ \\
\hline & TD 20 & $0.39 \mathrm{ab}$ & 0.03 & $69.1 \mathrm{abc}$ & $46.6 \mathrm{a}$ & $14.4 \mathrm{~cd}$ & $149.3 \mathrm{~d}$ & 0.0 & $106.6 \mathrm{~cd}$ \\
\hline & TD 40 & $0.39 \mathrm{ab}$ & 0.03 & $77.3 \mathrm{ab}$ & $53.6 \mathrm{a}$ & $18.2 \mathrm{a}$ & $163.9 \mathrm{~cd}$ & 0.0 & $232.7 \mathrm{a}$ \\
\hline & TD 60 & $0.35 \mathrm{abc}$ & 0.02 & $69.7 \mathrm{abc}$ & $39.5 \mathrm{a}$ & $14.4 \mathrm{~cd}$ & $150.2 \mathrm{~d}$ & 0.0 & $161.7 \mathrm{a}-\mathrm{d}$ \\
\hline & TD 80 & $0.41 \mathrm{a}$ & 0.02 & $38.1 \mathrm{c}$ & $44.9 \mathrm{a}$ & $17.5 \mathrm{ab}$ & $188.8 \mathrm{bc}$ & 0.0 & $171.5 \mathrm{abc}$ \\
\hline & INC 50 & $0.34 \mathrm{bc}$ & 0.02 & $47.9 \mathrm{bc}$ & $45.2 \mathrm{a}$ & $14.8 \mathrm{bcd}$ & $189.9 \mathrm{bc}$ & 0.0 & $133.8 \mathrm{bcd}$ \\
\hline & INC 100 & $0.39 \mathrm{ab}$ & 0.03 & 82.9 a & 57.9 & $15.1 \mathrm{bc}$ & 204.6 b & 0.0 & $188.6 \mathrm{ab}$ \\
\hline & INC 150 & $0.37 \mathrm{ab}$ & 0.03 & $67.5 \mathrm{abc}$ & 42.2 & $14.1 \mathrm{~cd}$ & $272.1 \mathrm{a}$ & 0.1 & $163.1 \mathrm{a}-\mathrm{d}$ \\
\hline & INC 200 & $0.37 \mathrm{ab}$ & 0.02 & $45.8 \mathrm{bc}$ & 39.9 & $13.3 \mathrm{~cd}$ & $250.4 \mathrm{a}$ & 0.0 & $104.5 \mathrm{~cd}$ \\
\hline & $\mathrm{MM}+\mathrm{Si}$ & $0.31 \mathrm{c}$ & 0.03 & $94.3 \mathrm{a}$ & 42.5 & $11.9 \mathrm{~d}$ & $194.7 \mathrm{~b}$ & 0.3 & $97.1 \mathrm{~d}$ \\
\hline \multirow{10}{*}{$\begin{array}{l}\text { Festival Light } \\
\text { Eye White } \\
\text { Shades }\end{array}$} & 0 & $0.40^{\mathrm{y}}$ & 0.07 & 107.6 & 46.6 & 24.8 & 145.4 & $2.29 \mathrm{a}$ & 591.1 \\
\hline & TD 20 & 0.48 & 0.08 & 253.4 & 56.6 & 31.5 & 129.8 & $0.128 \mathrm{~b}$ & 392.1 \\
\hline & TD 40 & 0.39 & 0.07 & 185.2 & 52.8 & 20.9 & 129.2 & $0.002 b$ & 235.6 \\
\hline & TD 60 & 1.01 & 0.16 & 308.0 & 84.1 & 133.8 & 156.9 & $0.191 \mathrm{~b}$ & 390.6 \\
\hline & TD 80 & 1.03 & 0.14 & 264.7 & 115.2 & 171.8 & 190.8 & $0.066 \mathrm{~b}$ & 566.7 \\
\hline & INC 50 & 0.42 & 0.07 & 223.7 & 57.9 & 14.4 & 165.3 & $2.12 \mathrm{a}$ & 649.9 \\
\hline & INC 100 & 0.38 & 0.12 & 263.2 & 51.8 & 20.3 & 161.9 & $2.23 \mathrm{a}$ & 736.4 \\
\hline & INC 150 & 0.32 & 0.07 & 172.1 & 43.9 & 11.6 & 161.8 & $0.103 \mathrm{~b}$ & 305.9 \\
\hline & INC 200 & 0.31 & 0.06 & 163.9 & 43.6 & 10.5 & 219.1 & $0.131 \mathrm{~b}$ & 291.1 \\
\hline & $\mathrm{MM}+\mathrm{Si}$ & 0.62 & 0.08 & 308.1 & 79.2 & 67.9 & 198.8 & $1.48 \mathrm{ab}$ & 742.7 \\
\hline \multirow{10}{*}{ Denver Daisy } & 0 & $0.41 \mathrm{~cd}$ & 0.02 & $x$ & 39.7 & $6.03 \mathrm{de}$ & $137.6 \mathrm{bc}$ & 0.002 & $97.9 \mathrm{c}$ \\
\hline & TD 20 & $0.49 \mathrm{abc}$ & 0.02 & $x$ & 40.4 & $8.0 \mathrm{~b}-\mathrm{e}$ & $131.7 \mathrm{bcd}$ & 0.002 & $130.9 \mathrm{bc}$ \\
\hline & TD 40 & $0.48 \mathrm{abc}$ & 0.02 & $x$ & 42.7 & $8.9 \mathrm{~b}-\mathrm{e}$ & $114.4 \mathrm{~d}$ & 0.002 & $134.5 \mathrm{bc}$ \\
\hline & TD 60 & $0.52 \mathrm{ab}$ & 0.04 & $x$ & 39.8 & $8.6 \mathrm{a}-\mathrm{d}$ & $125.7 \mathrm{~cd}$ & 0.003 & $132.6 \mathrm{bc}$ \\
\hline & TD 80 & $0.44 \mathrm{bcd}$ & 0.04 & $x$ & 33.1 & 7.5 cde & $129.5 \mathrm{~cd}$ & 0.462 & 91.7 c \\
\hline & INC 50 & $0.55 \mathrm{a}$ & 0.02 & $x$ & 44.2 & $10.9 \mathrm{ab}$ & $137.1 \mathrm{bc}$ & 0.533 & $209.2 \mathrm{ab}$ \\
\hline & INC 100 & $0.53 \mathrm{ab}$ & 0.03 & $x$ & 36.8 & $10.0 \mathrm{abc}$ & $152.1 \mathrm{~b}$ & 0.308 & $168.8 \mathrm{bc}$ \\
\hline & INC 150 & $0.48 \mathrm{abc}$ & 0.03 & $x$ & 37.6 & 7.6 cde & 208.7 a & 0.145 & $139.5 \mathrm{bc}$ \\
\hline & INC 200 & $0.50 \mathrm{abc}$ & 0.03 & $x$ & 43.6 & $11.4 \mathrm{a}$ & $203.8 \mathrm{a}$ & 0.575 & $285.2 \mathrm{a}$ \\
\hline & $\mathrm{MM}+\mathrm{Si}$ & $0.34 \mathrm{~d}$ & 0.01 & $x$ & 37.1 & $5.1 \mathrm{e}$ & $140.3 \mathrm{bc}$ & 0.002 & $105.9 \mathrm{bc}$ \\
\hline \multicolumn{2}{|c|}{ Optimum levels } & $0.2-0.8$ & $\mathrm{w}$ & $\mathrm{w}$ & $27-100$ & $5.0-30.0$ & $20-300$ & $0-5$ & $100-500$ \\
\hline
\end{tabular}

${ }^{\mathrm{z}}$ Top-dressed (TD) or incorporated (INC). ${ }^{\mathrm{y}}$ Means $(\mathrm{n}=6)$ with the same letter within the same column and within cultivars are not statistically significant at $p \leq 0.05$. ${ }^{\times}$Significant interactions between diatomaceous earth and irrigation reported in another table. ${ }^{\mathrm{w}}$ Optimum levels not reported.

Table 7. Analysis of variance for growth, flowering, leaf nutrient content, soil silica, and physiology of Gerbera jamesonii ‘Festival Light Eye White Shades' with application of diatomaceous earth (DE) and irrigation controlled with a tensiometer.

\begin{tabular}{|c|c|c|c|c|c|c|c|c|c|c|c|}
\hline Source & \multicolumn{2}{|c|}{$\begin{array}{l}\text { Height } \\
\text { (cm) }\end{array}$} & \multicolumn{2}{|c|}{$\begin{array}{l}\text { Width } \\
\text { (cm) }\end{array}$} & \multicolumn{2}{|c|}{$\begin{array}{l}\text { Shoot Dry } \\
\text { Weight } \\
\text { (g) }\end{array}$} & \multicolumn{2}{|c|}{$\begin{array}{l}\text { Stem Diameter } \\
(\mathrm{cm})\end{array}$} & \multicolumn{2}{|c|}{$\begin{array}{l}\text { Mean Flower } \\
\text { Number }\end{array}$} & $\begin{array}{c}\text { Flower } \\
\text { Diameter } \\
\text { (cm) }\end{array}$ \\
\hline DE Treatment & \multicolumn{2}{|c|}{$* \mathrm{z}$} & \multicolumn{2}{|c|}{ * } & \multicolumn{2}{|c|}{ * } & \multicolumn{2}{|c|}{ ns } & \multicolumn{2}{|c|}{ ns } & ns \\
\hline Irrigation & \multicolumn{2}{|c|}{ ns } & \multicolumn{2}{|c|}{$* *$} & \multicolumn{2}{|c|}{ ns } & \multicolumn{2}{|c|}{ ns } & \multicolumn{2}{|c|}{ ns } & ns \\
\hline $\begin{array}{l}\text { DE Treatment } \\
\times \text { Irrigation }\end{array}$ & \multicolumn{2}{|c|}{ ns } & \multicolumn{2}{|c|}{ Ns } & \multicolumn{2}{|c|}{ ns } & \multicolumn{2}{|c|}{ ns } & \multicolumn{2}{|c|}{ ns } & ns \\
\hline Source & $\begin{array}{c}\mathrm{N} \\
(\%)\end{array}$ & $\begin{array}{c}\mathbf{P} \\
(\%)\end{array}$ & $\begin{array}{c}S \\
(\%)\end{array}$ & $\underset{(\%)}{K}$ & $\begin{array}{l}\mathrm{Mg} \\
(\%)\end{array}$ & $\begin{array}{l}\text { Ca } \\
(\%)\end{array}$ & $\begin{array}{l}\mathrm{Na} \\
(\%)\end{array}$ & $\begin{array}{c}\mathrm{Si} \\
(\mathrm{ppm})\end{array}$ & $\begin{array}{c}\mathrm{Zn} \\
(\mathrm{ppm})\end{array}$ & $\underset{(p p m)}{\mathrm{Cu}}$ & $\begin{array}{c}\text { Fe } \\
(\mathrm{ppm})\end{array}$ \\
\hline DE Treatment & ns & ns & ns & ns & ns & ns & ns & ns & ns & ns & ns \\
\hline Irrigation & ns & ns & ns & ns & $*$ & $*$ & $* *$ & $*$ & ns & ns & ns \\
\hline $\begin{array}{l}\text { DE Treatment } \\
\times \text { Irrigation }\end{array}$ & ns & ns & ns & ns & ns & ns & ns & ns & ns & ns & ns \\
\hline Source & \multicolumn{8}{|c|}{ Transpiration } & \multicolumn{3}{|c|}{ Soil silica (ppm) } \\
\hline DE Treatment & \multicolumn{8}{|c|}{ ns } & \multicolumn{2}{|c|}{$* * * *$} & \\
\hline Irrigation & \multicolumn{6}{|c|}{$* * * *$} & & & \multicolumn{2}{|r|}{$*$} & \\
\hline $\begin{array}{l}\text { DE Treatment } \\
\times \text { Irrigation }\end{array}$ & \multicolumn{6}{|c|}{$* *$} & & & \multicolumn{2}{|r|}{$* *$} & \\
\hline
\end{tabular}

${ }^{\mathrm{z}} \mathrm{NS},{ }^{*}, * *, * *, * * * *$ indicates non-significant or significant at $p \leq 0.05,0.01$, and 0.001 , respectively. 
Table 8. Leaf nutrient content affected by irrigation, controlled by a tensiometer, across diatomaceous earth treatments in Dahlia $\times$ hybrida 'Dahlinova Montana', Gerbera jamesonii 'Festival Light Eye White Shades', and Rudbeckia hirta 'Denver Daisy'.

\begin{tabular}{|c|c|c|c|c|c|c|c|c|c|c|}
\hline Cultivar & $\begin{array}{l}\text { Application } \\
\text { and Rate }(g)^{z}\end{array}$ & $\underset{(\%)}{N}$ & $\begin{array}{c}P \\
(\%)\end{array}$ & $\begin{array}{c}\mathrm{S} \\
(\%)\end{array}$ & $\begin{array}{c}\mathrm{K} \\
(\%)\end{array}$ & $\begin{array}{l}\mathrm{Mg} \\
(\%)\end{array}$ & $\begin{array}{l}\mathrm{Ca} \\
(\%)\end{array}$ & $\begin{array}{l}\mathrm{Na} \\
(\%)\end{array}$ & $\begin{array}{c}\mathrm{Si} \\
(\mathrm{ppm})\end{array}$ & $\begin{array}{c}\mathrm{Zn} \\
(\mathrm{ppm})\end{array}$ \\
\hline Dahlinova & 10 & $3.73^{z}$ & 0.35 & 0.37 & 3.39 & 0.93 & 1.83 & 0.03 & 68.1 & 46.1 \\
\hline Montana & 20 & 3.79 & 0.33 & 0.35 & 3.52 & 0.94 & 1.89 & 0.03 & 67.2 & 44.1 \\
\hline Festival Light Eye & 10 & $2.89^{\mathrm{z}}$ & 0.27 & 0.41 & $3.01 \mathrm{~b}$ & 0.61 & $1.45 \mathrm{~b}$ & $0.07 \mathrm{~b}$ & 184.2 & 51.5 \\
\hline White Shades & 20 & 2.99 & 0.24 & 0.66 & $3.14 \mathrm{a}$ & 0.72 & $1.79 \mathrm{a}$ & $0.12 \mathrm{a}$ & 265.6 & 74.8 \\
\hline Denver Daisy & 10 & $2.88^{z}$ & 0.25 & $0.43 \mathrm{~b}$ & 3.48 & $1.03 \mathrm{~b}$ & $2.82 \mathrm{~b}$ & 0.03 & $\mathrm{y}$ & 37.5 \\
\hline \multicolumn{2}{|c|}{ Optimum levels ${ }^{x}$} & $2.50-4.50$ & $0.20-0.75$ & $0.25-1.00$ & $1.50-5.50$ & $0.25-1.00$ & $1.00-4.00$ & $\mathrm{w}$ & $\mathrm{w}$ & $27.0-100.0$ \\
\hline
\end{tabular}

${ }^{\mathrm{z}}$ Means $(\mathrm{n}=6)$ with the same letter within the same column and within cultivar are not statistically significant $p \leq 0.05$. ${ }^{\mathrm{y}}$ Significant interaction between diatomaceous earth treatment and irrigation. ${ }^{\mathrm{x}}$ According to Kalra (26). ${ }^{w}$ Optimum levels not reported.

A main effect of DE was seen for height, width, shoot dry weight, and nickel (Ni) content in the leaf tissue (Table 7). Height was greatest for $80 \mathrm{~g}$ TD plants, as well as 50, 100, and $150 \mathrm{~g}$ INC plants (Table 4). Width was greatest for TD plants at 20,40, and $60 \mathrm{~g}$, and all INC plants. Shoot dry weight was greatest for INC plants at 100 and $150 \mathrm{~g}$. Nickel was greatest for control plants, INC plants at 50 and $100 \mathrm{~g}$, as well as MM + Si plants (Table 6).

\subsection{Rudbeckia hirta 'Denver Daisy'}

A significant interaction of DE with irrigation was seen for leaf Si content (Table 9). Under the well-watered condition, silica in the leaf was greatest for 20 and $60 \mathrm{~g}$ TD plants, 50 and $100 \mathrm{~g}$ INC plants, as well as MM + Si plants (Table 2). Under the water-stressed condition, $\mathrm{Si}$ in the leaf was greatest for $60 \mathrm{~g}$ TD plants and MM + Si plants.

Table 9. Analysis of variance for growth, flowering, leaf nutrient content, soil silica, and physiology of Rudbeckia hirta 'Denver Daisy' after application of diatomaceous earth (DE) and irrigation controlled with a tensiometer.

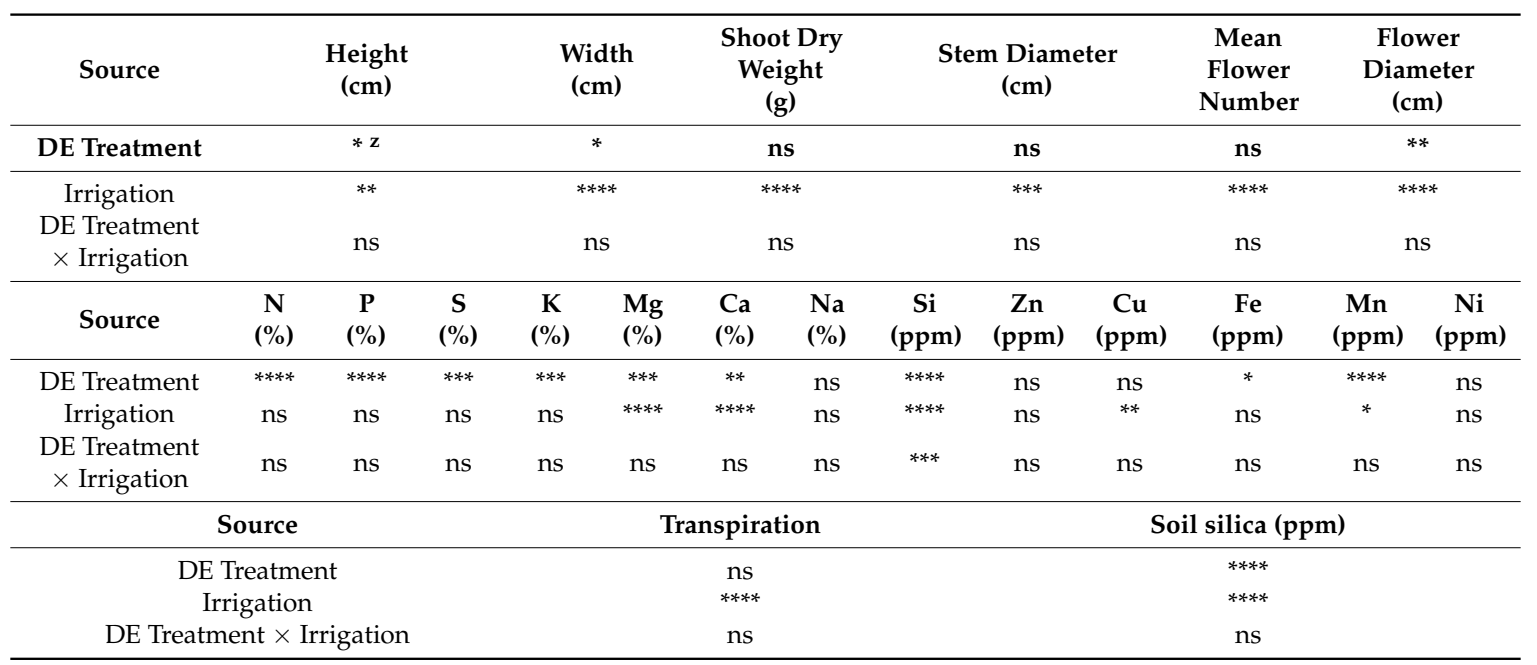

${ }^{\mathrm{z}} \mathrm{NS},{ }^{*}, * *, * * *, * * * *$ indicates non-significant or significant at $p \leq 0.05,0.01$, and 0.001 , respectively.

A significant effect of irrigation was seen for all growth and flower characteristics, as well as leaf nutrient content, transpiration, and soil Si (Table 9). Plants that were well-watered grew taller and wider, and had greater shoot dry weight, stem diameter, flower number, and flower diameter (Table 3). The nutrients $\mathrm{S}, \mathrm{Mg}, \mathrm{Ca}$, and $\mathrm{Mn}$ were greater in the leaf tissue of water-stressed plants, compared to well-watered plants (Table 8). Soil Si and transpiration were greater in plants under the water-stressed 
condition, compared to those that were well-watered (Table 10). A main effect of DE treatment was seen for height, width, and flower diameter (Table 9). Height was greatest for $60 \mathrm{~g}$ TD plants, $100 \mathrm{~g}$ INC plants, and MM + Si plants (Table 4). Width was greatest for all TD plants, INC plants at 50 and $100 \mathrm{~g}$, and MM + Si plants. Flower diameter was greatest for $50 \mathrm{~g}$ INC plants and MM + Si plants.

Table 10. Soil silica and transpiration of Rudbeckia hirta 'Denver Daisy' affected by irrigation, controlled by a tensiometer, averaged across silica treatments.

\begin{tabular}{cccc}
\hline Source & Irrigation Rate $\mathbf{( c b )}$ & Soil Si $\mathbf{( p p m )}$ & Transpiration \\
\hline Well-watered & 10 & $35.8 \mathrm{~b}^{\mathrm{z}}$ & $4.56 \mathrm{~b}$ \\
Water-stressed & 20 & $47.7 \mathrm{a}$ & $8.10 \mathrm{a}$ \\
\hline
\end{tabular}

\section{Discussion}

Amending the soilless substrate with varying rates of DE by top-dressing or by incorporating into the substrate increased plant height, width, shoot dry weight, stem diameter, and flower diameter in dahlia 'Dahlinova Montana', daisy 'Festival Light Eye White Shades', and black-eyed Susan 'Denver Daisy', in this study. Several other studies have reported similar benefits of supplemental Si on growth and flowering characteristics. Hwang et al. [21] reported that adding $200 \mathrm{mg} \mathrm{L}^{-1}$ of potassium (K) metasilicate increased plant height and shoot dry weight in cut roses. Stem quality was also improved in cut roses when Si was added to a recirculated nutrient solution in a closed hydroponic system [22]. Flower diameter of calibrachoa (Calibrachoa $\times$ hybrida Cerv.), fuchsia (Fuchsia hybrid hort. Ex Siebold $\&$ Voss), and petunia (Petunia $\times$ hybrida Vilm.) increased when supplemented with a weekly drench of K silicate at $100 \mathrm{mg} \mathrm{L}^{-1}$ [23]. Silica supplementation improved growth of two cultivars of French marigolds (Tagetes patula L.) by increasing stem diameter, shoots, and dry weights [24]. Growth and biomass parameters were increased in begonia (Begonia semperflorens Link et Otto) and pansy (Viola $\times$ wittrockiana Hort.) grown in vitro when supplemented with K silicate [25]. Savvas et al. [13] reported a greater percentage of flowers in hydroponically-grown gerbera (Gerbera jamesonii) supplemented with Si.

Amending the soilless substrate with DE increased nutrient content, despite being inert. Based on the analysis of Kalra [26], most nutrients were within the optimum range adequate for plant growth and levels greater than the maximum range were not considered excess or toxic. Nickel concentrations in dahlia 'Dahlinova Montana' were less than the minimum range (Table 4). However, these levels were not considered insufficient because often there are no symptoms to accurately determine Ni deficiency [27]. Epstein [1] has noted that the presence of Si does, in fact, affect absorption and translocation of several macro-nutrients and micro-nutrients. Early studies conducted by Fisher [28] reported that the addition of Si made P more available in barley (Hordeum vulgare L.). Mali and Aery [29] found that, in wheat (Tritium aestivum L.), potassium uptake was improved even at low concentrations of $\mathrm{Si}$ by H-ATPase being activated. Phosphorus and $\mathrm{K}$ are essential nutrients for flowering characteristics. Friedman et al. [30] conducted a study on sunflower (Helianthus annuus L.) and celosia (Celosia argentea L.), and reported that growth and flower parameters were increased when supplemented with an effluent containing high amounts of N, P, and other nutrients. Kamenidou et al. [7-9] also found an increase in $\mathrm{N}$ for sunflowers and gerbera, but most of the levels exceeded the optimum range. Nitrogen metabolism is a major factor in stem and leaf growth and too much can delay or prevent flowering. Calcium is part of the structure of cell walls and is necessary for cell growth and division. Ma and Takahashi [31] reported that there was an antagonistic effect between $\mathrm{Si}$ and $\mathrm{Ca}$ in rice, in which one can decrease the amount of the other. However, our study found the opposite effect, in which MM + Si increased Ca content in dahlia 'Dahlinova Montana' and 'Denver Daisy'. Kamenidou et al. [9] and Savvas et al. [13] also reported that supplemental Si increased $\mathrm{Ca}$ within gerbera. There was an increase in metals such as $\mathrm{Cu}, \mathrm{Fe}$, and $\mathrm{Mn}$ in dahlia 'Dahlinova Montana' and daisy 'Festival Light Eye White Shades', due to DE having trace amounts of these 
elements [18]. Silica levels in the leaf tissue and media for all the plants were observed in low amounts. Potentially, the plants could be classified as non-accumulators of $\mathrm{Si}(<0.5 \%)$ which has been reported for gerbera [32].

Amending the soilless substrate with varying methods and rates of DE showed mixed results on transpiration in all three species. Improvements in this physiological trait were mostly seen when $\mathrm{Si}$ (DE) supplementation interacted with irrigation. Kamenidou et al. [33] found that a foliar spray of $\mathrm{Na}$ silicate at $100 \mathrm{mg} \mathrm{L}^{-1}$ decreased transpiration in zinnias (Zinnia L.). Yoshida and Kitagishi [34] noted that the effects are related to $\mathrm{Si}$ being deposited in the cuticular layers of leaves, serving as a barrier which reduces the loss of water. A decrease in transpiration can benefit the floricultural market by improving quality and shelf life of cut flowers [35]. Considering the effect of irrigation, plants under the well-watered condition had greater growth and flowering, which was expected. However, $\mathrm{Si}$ is known to maintain the growth and flowering characteristics as well as nutrient levels in water-stressed plants. In Kentucky bluegrass (Poa pratensis L.), drought stress hindered physiological and quality attributes, but application of $\mathrm{Si}$ alleviated the adverse effects [36].

\section{Conclusions}

Several growth and flowering characteristics were improved, depending on the rate and application method, by application of DE. Benefits of DE included increased height, width, shoot dry weight, stem, and flower diameter. An increase in nutrients, such as $\mathrm{N}, \mathrm{P}, \mathrm{K}, \mathrm{Mg}$, and $\mathrm{Ca}$, was seen mostly for dahlia 'Dahlinova Montana' and black-eyed Susan 'Denver Daisy'. The adverse effects that typically occur under water-stressed conditions were alleviated and plant quality, as well as transpiration, was maintained in all three plants due to Si supplementation. Silicon is known to play an important role in cell membrane integrity, in which osmosis, photosynthesis, and transpiration all occur. Diatomaceous earth as supplemental Si was beneficial for plant growth, flowering, and nutrient content under both well-watered and water-stressed conditions. For growth and flower characteristics, $\mathrm{MM}+\mathrm{Si}$ was similar to the control (MM) with no added silica, and equivalent to most treatments with supplemental silica. To conclude, this research supports that DE, one of the many Si sources, is beneficial to plants; however, this is dependent upon species, Si rate, and the method of application. Benefits of DE include an increase in growth parameters, leaf nutrient content, and tolerance to stress, in which plant quality can be maintained. Future studies should further assess the use of DE on other crops and stress conditions.

Author Contributions: Conceptualization, B.D.; methodology, T.M.-I.; formal analysis, M.P.; investigation, T.M.-I.; writing—original draft preparation, T.M.-I.; writing—review and editing, B.D and N.M.; supervision, B.D.

Funding: This research was funded by ODAFF Specialty Block grant \#G00010135.

Acknowledgments: We thank Stephen Stanphill for helping with data collection and greenhouse management.

Conflicts of Interest: The authors declare no conflict of interest.

\section{References}

1. Epstein, E. The anomaly of silicon in plant biology. Proc. Natl. Acad. Sci. USA 1994, 91, 11-17. [CrossRef] [PubMed]

2. Ma, J.F.; Yamaji, N. Silicon uptake and accumulation in higher plants. Plant Sci. 2006, 11, 392-397. [CrossRef] [PubMed]

3. Chérif, M.; Asselin, A.; Bélanger, R.R. Defense responses induced by soluble silicon in cucumber roots infected by Pythium spp. Phytopathology 1994, 84, 236-242. [CrossRef]

4. Ma, J.F. Role of silicon in enhancing the resistance of plants to biotic and abiotic stresses. Soil Sci. Plant Nutr. 2004, 50, 11-18. [CrossRef]

5. Liang, Y.; Sun, W.; Zhu, Y.G.; Christie, P. Mechanisms of silicon-mediated alleviation of abiotic stresses in higher plants: A review. Environ. Pollut. 2006, 147, 422-428. [CrossRef] [PubMed]

6. Voogt, W.; Sonneveld, C. Silicon in Agriculture; Elsevier: Amsterdam, The Netherlands, 2011. 
7. Kamenidou, S.; Cavins, T.J.; Marek, S. Silicon supplements affect horticultural traits of greenhouse-produced ornamental sunflowers. HortScience 2008, 43, 236-239. [CrossRef]

8. Miyake, Y.; Takahashi, E. Silicon deficiency of tomato plant. Soil Sci. Plant Nutr. 1987, 24, 175-189. [CrossRef]

9. Kamenidou, S.; Cavins, T.J.; Marek, S. Silicon supplements affect floricultural quality traits and elemental nutrient concentrations of greenhouse produced gerbera. Sci. Hortic. 2010, 123, 390-394. [CrossRef]

10. De Kreij, C.; Voogt, W.; Baas, R. Nutrient solutions and water quality for soilless cultures. In Research Station for Floriculture and Glasshouse Vegetables Brochure; Naaldwijk Office: Naaldwijk, The Netherlands, 1999.

11. Reezi, S.; Babalar, M.; Kalantari, S. Silicon alleviates salt stress, decreases malondialdehyde content and affects petal color of salt stressed cut rose (Rosa xhybrida L.) Hot Lady. Afr. J. Biotechnol. 2009, 8, 1502-1508.

12. Carvalho-Zanao, M.P.; LAZ, J.; Barbosa, J.G.; Grossi, J.A.S.; Ávila, V.T. Yield and shelf life of Chrysanthemum in response to the silicon application. Hortic. Bras. 2012, 30, 403-408. [CrossRef]

13. Savvas, D.; Manos, G.; Kotsiras, A.; Souvaliotis, S. Effects of silicon and nutrient-induced salinity on yield, flower quality and nutrient uptake of gerbera grown in a closed hydroponic system. J. Appl. Bot. Food Qual. 2002, 76, 153-158.

14. Berthelsen, S.; Noble, A.D.; Kingston, G.; Hurney, A.; Rudd, A.; Garside, A. Improving Yield and ccs in Sugarcane through the Application of Silicon-Based Amendments; Final Report on SRDC Project CLW009. 2003. Available online: http:/ /hdl.handle.net/11079/12957 (accessed on 9 January 2019).

15. Muir, S. Plant-Available Silicon (Si) as A Protectant Against Fungal Diseases in Soil-Less Potting Media. Available online: https://www.ngia.com.au/Story?Action=View\&Story_id=1782 (accessed on 9 January 2019).

16. Savant, N.K.; Snyder, G.H.; Datnoff, L.E. Silicon management and sustainable rice production. Adv. Agron. 1996, 58, 151-199.

17. Meerow, A.W.; Broschat, T.K. Growth of Hibiscus in media amended with a ceramic diatomaceous earth granule and treated with a kelp extract. HortTechnology 1996, 6, 70-73. [CrossRef]

18. Pati, S.; Pal, B.; Badole, S.; Hazra, G.C.; Mandal, B. Effect of silicon fertilization on growth, yield, and nutrient uptake of rice. Commun. Soil Sci. Plant Anal. 2016, 47, 284-290. [CrossRef]

19. King, P.A.; Reddy, S. Soilless Growth Medium Including Soluble Silicon. Available online: https://patents. google.com/patent/US6074988A/en (accessed on 9 January 2019).

20. Jim, J.W.; Dolda, S.K.; Henderson, R.E. Soil silicon extractability with seven selected extractants in relation to colorimetric and ICP determination. Soil Sci. 2004, 169, 861-870.

21. Hwang, S.J.; Park, H.M.; Jeong, B.R. Effects of potassium silicate on the growth of miniature rose Pinnochio grown on rockwool and its cut flower quality. J. Jpn. Soc. Hortic. Sci. 2005, 74, 242-247. [CrossRef]

22. Ehret, D.L.; Menzies, J.G.; Helmer, T. Production and quality of greenhouse roses in recirculating nutrient systems. Sci. Hortic. 2005, 106, 103-113. [CrossRef]

23. Mattson, N.S.; Leatherwood, W.R. Potassium silicate drenches increase leaf silicon content and affect morphological traits of several floriculture crops grown in a peat-based substrate. HortScience 2010, 45, $43-47$. [CrossRef]

24. Sivanesan, I.; Son, M.S.; Lee, J.P.; Jeong, B.R. Effects of silicon growth of Tagetes patula L. 'Boy Orange' and 'Yellow Boy' seedlings cultured in an environment-controlled chamber. Propag. Ornam. Plants 2010, 10, 136-140.

25. Lim, M.Y.; Lee, E.J.; Jana, S.; Sivanesan, I.; Jeong, B.R. Effect of potassium silicate on growth and leaf epidermal characteristics of Begonia and Pansy grown in vitro. Hortic. Sci. Technol. 2012, 30, 579-585. [CrossRef]

26. Kalra, Y. Handbook of Reference Methods for Plant Analysis; CRC Press: Boston, MA, USA, 1998.

27. Buechel, T. Role of Nickel in Plant Culture. Available online: http:/ / www.pthorticulture.com/en/trainingcenter/role-of-nickel-in-plant-culture/ (accessed on 9 January 2019).

28. Fisher, R.A. A preliminary note on the effect of sodium silicate in increasing the yield of barley. J. Agric. Sci. 1929, 19, 132-139. [CrossRef]

29. Mali, M.; Avery, N.C. Influence of silicon on growth, relative water contents and uptake of silicon, calcium and potassium in wheat grown in nutrient solution. J. Plant Nutr. 2008, 31, 1867-1876. [CrossRef]

30. Friedman, H.; Bernstein, N.; Bruner, M.; Rot, I.; Ben-Noon, Z.; Zuriel, A.; Zuriel, R.; Finklestein, S.; Umiel, N.; Hagiladi, A. Application of secondary-treated effluents for cultivation of sunflower (Helianthus annus L.) and celosia (Celosia argentea L.) as cut flowers. Sci. Hortic. 2007, 115, 62-69. [CrossRef] 
31. Ma, J.F.; Takahashi, E. Interaction between calcium and silicon in water-cultured rice plants. Plant Soil 1993, 148, 107-113. [CrossRef]

32. Bloodnick, E. Role of Silicon in Plant Culture. Available online: http://www.pthorticulture.com/en/ training-center / role-of-silicon-in-plant-culture/ (accessed on 9 January 2019).

33. Kamenidou, S.; Cavins, T.J.; Marek, S. Evaluation of silicon as a nutritional supplement for greenhouse zinnia production. Sci. Hortic. 2009, 119, 297-301. [CrossRef]

34. Yoshida, S.; Ohnishi, Y.; Kitagishi, K. Chemical forms, mobility and deposition of silicon in rice plant. Soil Sci. Plant Nutr. 1962, 8, 15-21. [CrossRef]

35. Jana, S.; Jeong, B.R. Silicon: The most under-appreciated element in horticultural crops. Hortic. Res. 2014, 4, 1-19.

36. Saud, S.; Li, X.; Chen, Y.; Zhang, L.; Fahad, S.; Hussain, S.; Sadiq, A.; Chen, Y. Silicon application increases drought tolerance of Kentucky bluegrass by improving plant water relations and morphophysiological function. Sci. World J. 2014, 23, 17647-17655. [CrossRef] [PubMed]

(C) 2019 by the authors. Licensee MDPI, Basel, Switzerland. This article is an open access article distributed under the terms and conditions of the Creative Commons Attribution (CC BY) license (http://creativecommons.org/licenses/by/4.0/). 